\title{
Une campagne d'opinion contre Marie-Joseph Chénier en floréal an III ou la culture révolutionnaire en procès
}

An opinion compaign against Marie-Joseph Chénier in floreal an III, or the Revolutionary culture on trial

\section{Gauthier Ambrus}

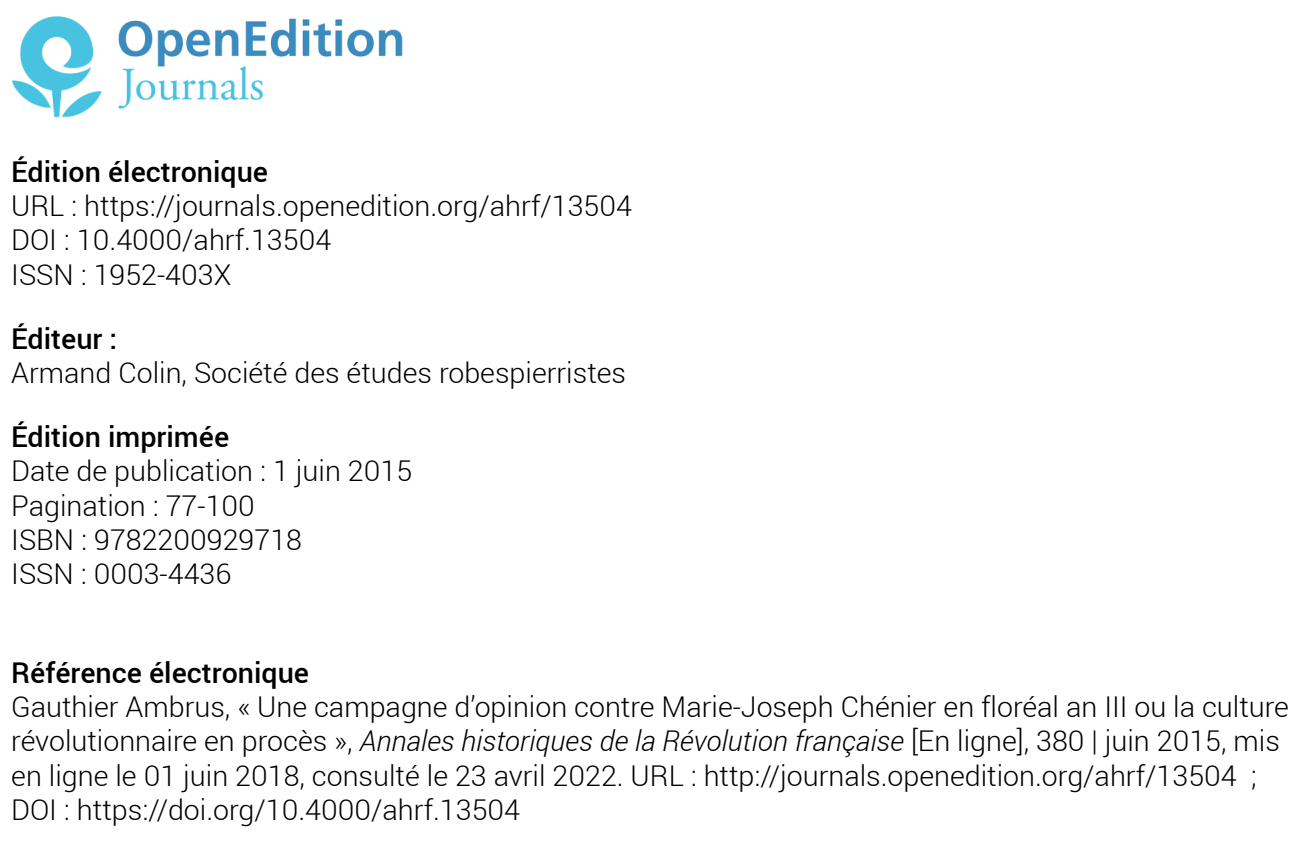




\title{
UNE CAMPAGNE D'OPINION CONTRE MARIE-JOSEPH CHÉNIER EN FLORÉAL AN III OU LA CULTURE RÉVOLUTIONNAIRE EN PROCÈS
}

\author{
Gauthier AMBRUS
}

\begin{abstract}
Le printemps 1795 marque un tournant dans la politique suivie jusque-là par la Convention thermidorienne qui, après la répression des émeutes de germinal, se retourne contre la " réaction ". Cette nouvelle impulsion républicaine s'incarne dans un projet de loi que Marie-Joseph Chénier fait voter au nom des Comités le 12 floréal an III. Parmi les mesures qu'il prévoit, la plus éclatante vise à modérer la presse contre-révolutionnaire, dont on redoute l'influence grandissante sur l'opinion. C'est reconnaître la centralité des publicistes dans le champ politique ouvert par le 9 Thermidor, au détriment de la Convention elle-même. L'intérêt de l'épisode ne réside pas seulement dans sa valeur clarificatrice. La loi déchaîne contre son rapporteur une formidable campagne d'opinion. En tirant prétexte de la carrière littéraire de Chénier, la presse réactionnaire intente un véritable procès à la culture révolutionnaire et à la notion d'« esprit public » qui la sous-tend.
\end{abstract}

Mots-clés : Chénier (Marie-Joseph), Thermidor, réaction, presse, esprit public, théâtre

\section{Le tournant républicain de mai 1795}

Au lendemain du 9 Thermidor, la chute de Robespierre est apparue comme la fin d'un régime d'oppression des esprits tout autant que des corps ${ }^{1}$.

(1) Voir les analyses d'Alma SöDERHJELM, Le régime de la presse pendant la Révolution

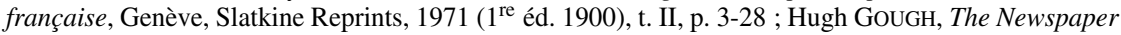
Press in the French Revolution, Londres, Routledge,1988, p. 120 sq. ; Bronislaw BACZKO, Comment sortir de la Terreur. Thermidor et la Révolution, Paris, Gallimard, 1989, p. 116-135 ; Carla HESSE, 
Elle marquait non seulement le retour de la liberté d'expression, mais aussi l'épuisement de la propagande républicaine : avec le gouvernement montagnard s'affaiblissait l'ambition de diriger l'esprit public. L'émancipation de l'opinion hors de l'influence jacobine, et donc sa parcellisation, semblait signer le crépuscule de la culture révolutionnaire et de son désir d'unité ${ }^{2}$. Le premier frein à ce mouvement devait nécessairement soulever une forte réaction, en particulier du côté des journalistes. C'est bien ce qui advint au printemps 1795, à l'occasion d'un ambitieux projet de décret présenté à la Convention par Marie-Joseph Chénier le 12 floréal an III ( $1^{\mathrm{er}}$ mai 1795), au nom des trois principaux comités de gouvernement ${ }^{3}$. Un mois après la répression des émeutes sans-culotte du 12 Germinal, les mesures stipulées par le texte ${ }^{4}$ opéraient un net changement de cap dans la politique thermidorienne de démontage du legs montagnard, pour tenter de contrecarrer la montée en puissance de la « réaction royaliste » : bannissement à perpétuité des émigrés et des condamnés à la déportation qui seront surpris sur le territoire de la République (et à la mort en cas d'infraction); encadrement plus strict du désarmement de l'ancien personnel terroriste ; traduction devant les tribunaux criminels des « individus qui, par leurs écrits ou leurs discours séditieux, auront provoqué l'avilissement de la Représentation nationale, ou le retour de la royauté », accusation pour laquelle ils encourent le bannissement à perpétuité ; mesures pour « diriger les écoles, les théâtres, et généralement les arts et les sciences vers le but unique des travaux de la Convention nationale : celui d'affermir la République »; enfin, rapport mensuel du Comité de salut public à la Convention «sur l'état de l'esprit public ». Alors que les dispositions antérieures sur la sécurité publique - comme lors du vote de la « loi de grande police » présentée par Sieyès le $1^{\text {er }}$ germinal an III - mettaient sur un même plan périls royaliste et jacobin (mais en visant surtout les seconds), les Comités pointaient donc désormais

Publishing and Cultural Politics in Revolutionary Paris, 1789-1810, Berkeley, University of California Press, 1991, p. 136-156 ; Raymonde MONNIER, L'Espace public démocratique. Essai sur l'opinion à Paris, de la Révolution au Directoire, Paris, Kimé, 1992, p. 189-205 ; Sergio LuZZATTO, L'Automne de la Révolution. Luttes et cultures politiques dans la France thermidorienne, trad. Simone CARPENTARIMEssina, Paris, H. Champion, 2001 (1 ${ }^{\text {re }}$ éd. 1994), p. 75-123. L'analyse de Sergio Luzzatto montre comment la conquête de « l'opinion » par les différents acteurs politiques devient l'enjeu capital des premiers mois de l'an III. Derrière ce terme, ce sont évidemment des catégories et des réalités bien différentes qui s'affrontent : « opinion du peuple » pour les derniers jacobins, opinion de la nation pour la majorité de la Convention thermidorienne, opinion de la «classe éclairée » et des « honnêtes gens » pour la presse anti-révolutionnaire. Cette dernière définition s'impose peu à peu.

(2) Voir Mona Ozouf, « Esprit public », Dictionnaire critique de la Révolution française, Idées, François Furet et Mona Ozouf (dir.), Paris, Flammarion, 1992 (1 ${ }^{\text {re }}$ éd. 1988), p. 165.

(3) Les Comités de salut public, de sûreté générale et de législation.

(4) Le Moniteur, 15 et 16 floréal an III. 
la contre-Révolution comme l'adversaire le plus dangereux. Il faut y voir l'une des premières manifestations de la naissance d'un parti du « centre $»^{5}$, destiné à donner une majorité républicaine à l'Assemblée, qui soit apte à la garantir aussi bien contre le mécontentement des sans-culottes que contre l'influence de la réaction (présente dans les sections et dans l'opinion modérée, avec des relais parmi les députés). Certes, cette brève résurrection du gouvernement révolutionnaire n'aura pas de grands effets, si ce n'est en faveur des jacobins arrêtés ou persécutés dans le Midi. Mais elle dut être perçue comme une manière de peser dans les débats en cours sur la nouvelle constitution. Enfin, la loi anticipe de fait des mesures d'exception (ou de préservation) comme le décret des deux-tiers et celui du 2 brumaire an IV, ou encore l'amnistie des délits liés à la Révolution (et exclus du code pénal) que la Convention votera le 4 brumaire an IV avant sa dissolution. Plus lointainement, elle trace les lignes majeures de la politique autoritaire suivie par le pouvoir républicain au cours du Directoire, marquée par une hésitation constitutive entre régime libéral et dirigisme révolutionnaire ${ }^{6}$.

L'article de loi sur la presse donne lieu à un débat particulièrement vif lorsqu'il est discuté à la Convention. Ne réactivait-il pas les législations révolutionnaires sur la liberté d'opinion, tout en adoucissant les peines ${ }^{7}$ ? (La loi Sieyès contenait déjà un article similaire, mais sa portée pénale était atténuée et, surtout, il n'était pas apparu comme une menace directe pour la presse contre-révolutionnaire, du fait de son contexte anti-jacobin). Les Comités eux-mêmes étaient partagés, comme le révèle Tallien, qui menace de démissionner du Comité de salut public si le texte est adopté : il $\mathrm{y}$ voit un grave attentat contre la liberté publique, faisant craindre un retour

(5) Pour situer cette notion dans l'histoire des idées politiques de la Révolution, voir Pierre SERNA, La République des Girouettes (1789-1815 et au-delà). Une anomalie politique : la France de l'extrême-centre, Paris, Champ Vallon, 2005, ainsi que Bernard GAINOT, « Benjamin Constant et le Cercle constitutionnel de 1797 : la modération impossible », $A H R F, \mathrm{n}^{\circ} 357$, juillet-septembre 2009, p. 103-118.

(6) Lynn Hunt, David LANSKY et Paul HAnson, « The Failure of the Liberal Republic in France, 1795-1799», Journal of Modern History, n51, 1979, p. 734-759 ; Isser WOLOCH, « "Republican Institutions", 1797-1799 », dans Colin LuCAS (dir.), The French Revolution and the Creation of Modern Political Culture, t. II, The Political Culture of the French Revolution, Oxford, Pergamon, 1988, p. 371-387 ; Howard G. BROwn, Ending the French Revolution. Violence, Justice, and Repression from Terror to Napoleon, Charlottesville and London, University of Virginia Press, 2006 (en particulier p. 23-46) ; Andrew JAINCHILL, Reimagining Politics after the Terror. The Republican Origins of French Liberalism, Ithaca and London, Cornell University Press, 2008.

(7) Les lois votées les 4 décembre 1792, 29 mars 1793, 17 septembre 1793 et 22 prairial an II prévoyaient toutes la peine capitale (voir sur la question Charles WALTON, Policing Public Opinion in the French Revolution. The Culture of Calumny and the Problem of Free Speech, New York, Oxford University Press, 2009, qui passe rapidement sur la loi du 12 floréal an III). Celle du $1^{\text {er }}$ germinal an III se démarquait déjà en remplaçant la mort par la déportation, ce qui n'empêcha pas des accusations de retour à la Terreur. 
de la Terreur. Si le texte est finalement voté, les réactions à l'extérieur de l'Assemblée seront d'une tout autre mesure. La presse hostile à la Révolution va en effet se déchaîner avec une violence sans précédent à l'égard de la Convention thermidorienne. Fait remarquable, sa campagne visera exclusivement la personnalité du rapporteur.

C'est à un poète à peine immiscé dans les affaires publiques qu'il était revenu d'assumer le décret devant l'opinion. Chénier avait intégré le Comité de sûreté générale lors du renouvellement partiel du 15 germinal $^{8}$. Membre timide du groupe montagnard jusqu'au 9 Thermidor, il s'était surtout cantonné dans ses travaux pour le Comité d'instruction publique. Son souci de l'esprit public n'était donc peut-être pas étranger à la préparation de la loi du 12 floréal. Aux mesures positives (théâtres, fêtes décadaires) mises en sommeil depuis la fin de la Terreur, elle substituait la censure et l'encadrement policier. Les adversaires de la « loi Chénier» en donneront immédiatement une lecture ad personam, en la mettant au compte du rapporteur. À tort ou à raison, l'opinion va ainsi tenir Chénier pour le principal responsable du virage républicain donné au gouvernement (même si d'autres, comme Sieyès, sont sans doute à manœuvrer dans l'ombre ${ }^{9}$ ). Probablement parce qu'il y avait des raisons de le supposer derrière l'article sur la censure, qui apparaissait comme le signe le plus évident de ce retour à l'ordre révolutionnaire.

Même si la mesure restera sans réelles conséquences, la polémique qu'elle entraîne mérite toutefois de retenir l'attention : elle marque non seulement la première charge de l'opinion libérale contre une République qui se place au-dessus des lois, mais aussi le dernier « procès » thermidorien,

(8) Sur l'entrée de Chénier dans la politique thermidorienne, voir Gauthier AMBRUS, « MarieJoseph Chénier et les Girondins de l'an III », Cahiers Roucher-André Chénier, n³3, 2013, p. 131-157, et pour les années antérieures, Alfred J. BINGHAM, Marie-Joseph Chénier's Early Life and Political Ideas (1789-1794), New York, 1939.

(9) Voir ce que rapporte Mallet du Pan en août 1795 : « Le parti Girondin Républicain tient sa cabale principale chez Julie Talma. Sieyès, Chénier, Louvet, Guyomard, Bailleul décident là le destin de l'État. » (Correspondance inédite de Mallet du Pan avec la cour de Vienne, Paris, E. Plon, Nourrit et $C^{\text {ie }}, 1884$, t. I, p. 269). Thibaudeau rapporte que Tallien et Fréron considéraient alors Chénier comme un des « séides » de Sieyès (Antoine Clair THIBAUdEAU, Mémoires sur la Convention et le Directoire, éd. François PASCAL, Paris, SPM, 2007, p. 275). 
mené cette fois contre la culture institutionnelle issue de la Révolution ${ }^{10}$ et l'idée d'esprit public qui lui est attachée. Elle avait connu un début d'ébranlement avec l'échec des projets de fêtes décadaires durant l'hiver précédent et l'adoption, le 19 pluviôse an III, du décret prévoyant qu'aucun citoyen ne pourrait être l'objet d'honneurs publics avant un délai posthume de dix ans, ce qui avait entraîné l'exclusion de Le Peletier et de Marat du Panthéon, ainsi que le retrait de tous les bustes des martyrs de $1793^{11}$. Mais le «procès » n'est pas clos aux yeux de l'opinion. Le Courrier républicain écrit à ce sujet : "Je comprends fort bien comment on peut dépanthéoniser Marat, mais je ne concevrai jamais comment on pourra démaratiser le Panthéon. $»^{12}$. En réalité, le phénomène touche au cœur même de l'entreprise républicaine et de ses contradictions, en débordant le cadre de la période thermidorienne : avatar de la rivalité entre la Convention et les sections, il s'inscrit dans une continuité certaine - mais à interroger avec la « désansculottisation » mise en œuvre par le Comité de salut public au printemps 1794, qui avait vu notamment la suppression du culte de Marat $^{13}$. Le processus s'est accéléré au lendemain du 9 Thermidor, avec l'interdiction par la Convention des surnoms révolutionnaires le 6 fructidor an II et la réorganisation des fêtes révolutionnaires autour d'un modèle où le peuple est tenu à distance ${ }^{14}$. Pour la Convention de l'an III, l'enjeu est de maintenir une culture révolutionnaire institutionnalisée qui survive à sa « dépopularisation ». La nécessité en est d'autant plus grande devant les assauts de la réaction. C'est donc dans ce cadre qu'il faut comprendre les mesures d'« esprit public » prévues dans la loi du 12 floréal an III.

(10) Elle avait été jusque-là contestée de façon ponctuelle (surtout dans sa composante sans-culotte) et plutôt mesurée. La presse de droite s'attachait en premier lieu à défendre la « liberté de culte », en l'occurrence celle de l'Église. Le dépouillement de la Quotidienne dans les mois qui précèdent le rapport de Chénier montre une intensification des critiques, y compris à l'égard des institutions nées après le 9 Thermidor. Leur virulence se précise avec la répression des émeutes de Germinal, qui libère les publicistes de leur prudence ordinaire. Voici les thèmes abordés à partir du 10 février 1795 : le calendrier républicain ( 25 ventôse, 26 et 29 germinal, 13 floréal), les prénoms révolutionnaires ( 5 germinal), les symboles et les innovations linguistiques de la Révolution (24, 25 et 26 germinal), l'École normale (4 floréal), les écoles primaires (12 floréal), les fondements philosophiques de la République (4, 5 et 9 floréal).

(11) Le Moniteur, 22 et 23 pluviôse an III (10 et février 1795). La Convention conserve seulement son buste de Brutus.

(12) Cité par Joseph de MAISTRE, Considérations sur la France [1797], Jean TuLARD (éd.), Paris, Éditions Garnier Frères, 1980, p. 104.

(13) Voir Serge BIANCHI, La Révolution culturelle de l'an II. Élites et peuple (1789-1799), Paris, Aubier, 1982, p. 243-259.

(14) Voir Mona Ozouf, La Fête révolutionnaire (1789-1799), Paris, Gallimard, 1976, p. 125148. 
La presse réactionnaire, marchant d'un bloc ${ }^{15}$, va réagir comme si elle se trouvait confrontée à un ultime coup de « queue » du robespierrisme, dérisoire cette fois, puisqu'il serait le fait d'un homme de lettres qui tente, envers et contre tout, de maintenir un état d'esprit condamné, une « terreur » intellectuelle, auxquels il devrait toute son existence, littéraire hier, politique aujourd' hui. L'épisode aura du poids, puisqu'il entachera sans doute définitivement la réputation de Chénier dramaturge ${ }^{16}$. Il contribuera ainsi à discréditer la littérature révolutionnaire, dont ce dernier est l'un des représentants éminents, comme poète tragique et comme auteur de la quasi-totalité des grands hymnes chantés entre 1791 et 1794, en imposant l'idée qu'elle s'est irrémédiablement compromise avec les dérapages de la politique. La mémoire collective en retiendra surtout les imputations de fratricide lancées contre le frère cadet d'André Chénier, aux dépens du sens politique qu'elles revêtirent en l'an III. Ainsi prenait naissance une de ces "légendes noires » à l'encontre des anciennes figures du jacobinisme, auxquelles la période thermidorienne était si propice ${ }^{17}$. À cette différence près que Chénier n'ayant guère de sang sur les mains, il fallait aller en chercher dans ses écrits. Passé de la mouvance girondine à une position inconfortable de montagnard « modéré », l'auteur de Charles IX s'était tenu volontairement à l'écart de toute influence politique avant le 9 Thermidor, refusant de siéger au Comité de sûreté générale en octobre 1792, puis déclinant une mission dans le Sud-Ouest en juillet 1793. Entré en Révolution par le biais de la littérature, il entendait visiblement s'y maintenir en concentrant son action sur l'instruction publique. C'est donc d'abord pour ses travaux au Comité d'instruction publique, ses hymnes et, dans une moindre mesure, son théâtre que Chénier a vu son nom associé

(15) Selon le témoignage de Lacretelle jeune, les journalistes hostiles à la Révolution, malgré leurs divergences, formaient un groupe d'influence bien organisé qui avait pour objectif de démanteler les lois révolutionnaires. Ils se réunissaient une fois par semaine dans un restaurant place du Louvre, autour de l'ancien censeur royal Suard. On y décidait de la marche à suivre pour gagner l'opinion et les députés sur tel ou tel point d'actualité (Charles de LACRETELLE, Dix années d'épreuves pendant la Révolution, Paris, 1842, p. 203-208). Voir Jacques GODECHOT, « La presse française sous la Révolution et l'Empire », dans Histoire générale de la presse française, Paris, PUF, 1969, t. I, p. 524-537 et Jeremy D. POPKIN, The Right-Wing Press in France, 1792-1800, Chapell Hill, The University of North Carolina Press, 1980, p. 29-53.

(16) Le destin littéraire des Chénier au XIX ${ }^{\mathrm{e}}$ siècle a été esquissé par Jean M. GouLEMOT et Jean-Jacques TAtin-Gourier, André Chénier. Poésie et politique, Paris, Minerve, 2005, ch. VI.

(17) Pour des études de cas notables, voir Michel BIARD, Collot d'Herbois : légendes noires et Révolution, Lyon, Presses universitaires de Lyon, 1995, p. 181-190 et Bronislaw BACZKO, « Comment est fait un tyran? Thermidor et la légende noire de Robespierre », dans Politiques de la Révolution française, Paris, Gallimard, 2008, p. 133-164. Au-delà d'une simple logique de règlement de comptes, Pierre Serna insiste sur la dimension proprement politique de la violence verbale déployée par la réaction thermidorienne, qui vise à discréditer l'adversaire (op. cit., p. 398-399). 
à celui du gouvernement montagnard, en dépit d'une position politique souvent en décalage avec les attentes jacobines, qui lui valut quelques contrariétés et la crainte d'une proscription au printemps 1794.

\section{Une intrigue politique : l'affaire Lefortier}

Qu'on ait exclusivement attribué le décret sur la presse à Chénier (comme Tallien le sous-entend à la Convention) ne manque pas de surprendre a priori. L'explication est à chercher dans les événements des semaines précédentes, dont l'un en particulier révèle les tensions secrètes qui agitent alors les rangs thermidoriens. À y bien regarder, les hostilités avaient en effet commencé quelques jours plus tôt. Les journalistes de l'opposition étaient en alerte depuis un certain temps sur les risques de censure. La menace planait sur les deux extrêmes du champ politique : les autorités avaient fait incarcérer Babeuf en pluviôse et Richer-Serizy en germinal ${ }^{18}$. Mais un épisode moins connu causa beaucoup d'émoi. Le rédacteur de la Correspondance politique de Paris et des départements, Jean-François Lefortier, avait été arrêté le 21 germinal sur ordre du Comité de sûreté générale ${ }^{19}$ et était depuis resté détenu sans jugement. On l'accusait de propagande royaliste pour avoir reproduit un article de la presse étrangère qui s'apitoyait sur le sort de Louis XVI ${ }^{20}$. Or l'affaire allait se révéler plus compliquée. Fréron, dans l'Orateur du peuple des $1^{\text {er }}$ et 5 floréal, prenait la défense du journaliste emprisonné. N'avait-il pas lui-même réclamé la « liberté illimitée » de la presse dès août 1794 ? Il faut dire également que son propre journal employait comme rédacteur un proche de Lefortier, Jean-Joseph Dussault ${ }^{21}$, ce qui suggère une accointance entre les deux périodiques. Dénoncé à son tour aux Comités, Fréron ne manqua pas de remarquer dans son numéro du lendemain que le même Lefortier avait publié plusieurs morceaux dirigés contre les membres du

(18) La Quotidienne, 30 ventôse an III.

(19) Albert SOBOUL et Raymonde MONNIER, Répertoire du personnel sectionnaire parisien en l'an II, Paris, Publications de la Sorbonne, 1985, p. 109.

(20) Alma SöDERHJELM, op. cit., t. II, p. 32. La Quotidienne rend compte de l'épisode dans son numéro du 25 germinal an III (14 avril 1795) : «L'auteur de la correspondance politique vient d'être arrêté par ordre du comité de sûreté générale. Il est accusé d'avoir donné, dans son journal, des éloges à Louis XVI et de regrets à la monarchie. » Lefortier sera remis en liberté fin floréal ( $L a$ Quotidienne, $1^{\mathrm{er}}$ prairial an III).

(21) Albert MATHIEZ, La Réaction thermidorienne, Paris, Armand Colin, 1929, p. 36-37. Dussault et Lefortier rédigeaient de concert la Correspondance politique de Paris et des départements en 1793-1794 (Jeremy D. PoPKIN, « The Royalist Press in the Reign of Terror », Journal of Modern History, vol. 51, n4 1979, p. 697-698). Il est fort possible que leur collaboration se soit poursuivie au printemps 1795. Dussault polémiquera contre Chénier jusque sous l'Empire. 
gouvernement, en particulier contre Chénier ${ }^{22}$. Il n'en fallait pas plus pour supposer que le malheureux journaliste avait été victime d'un règlement de comptes. De quoi s'agissait-il exactement ? Les numéros concernés n'ont pas été conservés ${ }^{23}$, mais l'Orateur du peuple parle d' « épigrammes », de « sarcasmes » et de «badinages » ${ }^{24}$. Plus précises, les Annales patriotiques et littéraires laisseront entendre que le journaliste se moquait de la dernière tragédie en date de Chénier, Timoléon, et peut-être aussi qu'il l'attaquait sur ses rapports avec André. Interdite par les Comités au printemps 1794 et représentée avec succès en septembre suivant, la pièce montrait un héros républicain, Timoléon, autorisant le meurtre de son frère Timophane, qui aspirait à la tyrannie ${ }^{25}$. Le sujet se prêtait aisément à une exploitation polémique si on le rapprochait de l'exécution d'André Chénier juste avant le 9 Thermidor. Les frères Chénier s'étaient engagés dans des partis adverses dès les premières années de la Révolution : André était devenu l'une des plumes les plus brillantes des feuillants, tandis que Marie-Joseph avait mis sa célébrité de dramaturge patriote au service des jacobins. Ils s'étaient publiquement affrontés dans la presse au printemps 1792, en un combat politique dont les suites indirectes s'étaient révélées fatales à l'aîné. À travers le poète dramatique, c'était donc son passé révolutionnaire d'ultra supposé qu'on visait ${ }^{26}$.

Mais pourquoi s'en prendre précisément à Chénier ? Tout indique que le choix de la cible correspondait à un dessein délibéré, qui dépassait le seul Lefortier. L'usage de l'épigramme à des fins politiques répondait à une stratégie affichée de la presse contre-révolutionnaire pour abattre une figure publique aux yeux de l'opinion, substituant ainsi un tort moral à la violence physique qui est l'apanage des terroristes ${ }^{27}$. Jusqu'alors, Chénier avait plutôt fait figure d'allié des réacteurs. Son intervention du 18 ventôse à la Convention n'avait-elle pas conduit au retour des derniers députés

(22) L'Orateur du peuple, 5 floréal an III.

(23) Les exemplaires de la Correspondance politique disponibles à la $\mathrm{BnF}$ vont du 2 vendémiaire au 12 nivôse an III. Les Archives nationales possèdent une série de numéros postérieurs (27 messidor-12 thermidor an III), qui ne contiennent aucune allusion directe à Chénier, même si la ligne du périodique ne s'est pas modifiée.

(24) L'Orateur du peuple, op. cit.

(25) Voir Marie-Joseph CHÉNIER, Théâtre, Gauthier AMBrus et François JACOB (éd.), Paris, GF-Flammarion, 2002, p. 42-52.

(26) C'est également ce qui ressort d'un pamphlet anonyme paru à la même période (Timoléon à Timophane, ou le Tyran dévoilé, s.l.n.d.), où Chénier n'est pas identifié à Timoléon mais aux ambitions tyranniques de Timophane, ce qui prouve que le sens de l'œuvre importait moins que le parti à en tirer.

(27) « Aux poignards, aux couteaux des bourreaux, nous opposons le sarcasme et l'épigramme ; on croit avoir tout fait pour la vengeance et la tranquillité du peuple, lorsqu'on a hué dans un spectacle le personnage fabuleux d'un brigand révolutionnaire. » (La Quotidienne, 2 floréal an III). 
girondins encore proscrits ? On serait bien en peine de trouver la moindre allusion négative à son égard dans l'ensemble de la presse thermidorienne « de droite » avant le printemps 1795 . Tout au contraire, la reprise de sa tragédie de Fénelon, qui défend la tolérance politique en empruntant le langage de la religion, fut saluée comme un signe heureux du changement de disposition des esprits ${ }^{28}$. Est-ce un hasard si les attaques de Lefortier suivirent immédiatement les fermes réquisitoires contre la « réaction » que Chénier prononça à l'Assemblée les 10 et 13 germinal, où il s'en prenait déjà à l'audace anti-révolutionnaire de certains périodiques ? Il semble être en effet le premier thermidorien à avoir systématisé l'usage politique du mot. Un tel retournement chez un proche du gouvernement ne sera certainement pas passé inaperçu. La proximité de Lefortier et Dussault avec Fréron et sans doute Tallien ${ }^{29}$ laisse penser que les escarmouches contre Chénier trouvaient leur origine dans les dissensions internes de la majorité thermidorienne ${ }^{30}$, déchirée entre ses ailes républicaine et contre-révolutionnaire. De fait, le décret sur la liberté de la presse entraînait l'annulation de cette zone grise où se côtoyaient hommes politiques et publicistes de la réaction. Fréron et Tallien en étaient les noms les plus éclatants, puisqu'ils nourrissaient leur influence à la Convention des échos reçus par leurs périodiques, où œuvraient de véritables têtes de pont du royalisme, comme Méhée ou Dussault (et Lefortier ?). Les deux députés seront les premiers, nous l'avons vu, à prendre la défense du journaliste incarcéré et à s'opposer aux mesures de Chénier, et ce, avant même qu'elles soient votées. Mi-floréal, la presse royaliste (du moins officieusement) cherchera plus que jamais à diviser le nouveau front républicain ${ }^{31}$. Et Marie-Joseph Chénier, fragilisé

(28) Correspondance politique de Paris et des départements, 15 frimaire an III.

(29) Chénier déclarera dans les derniers jours de la Convention qu'il avait soupçonné Tallien de collusion avec les royalistes à cause de ses bons rapports avec la presse contre-révolutionnaire (Moniteur, 7 brumaire an IV). Plusieurs figures de ce milieu fréquentaient notoirement le salon de $\mathrm{M}^{\mathrm{me}}$ Tallien (Charles de LACRETELLE, op. cit., p. 8-11 et 197-197). Le parcours de certains journalistes royalistes était fort sinueux et avait croisé celui de Tallien et Fréron avant Thermidor. Les rédacteurs de la Correspondance politique, Dussault et Lefortier, engagés dans la contre-Révolution depuis le printemps 1793, avaient curieusement bénéficié de l'indulgence des autorités de l'an II, et le premier soutint du reste plusieurs mesures contre les sans-culottes. Mais, dès messidor, leurs articles auraient laissé transparaître une certaine connivence avec les instigateurs du 9 Thermidor (Jeremy D. POPKIN, art. cit., p. 697-698).

(30) On en a une parfaite illustration dans un pamphlet publié quelques semaines plus tard par Dussault, à l'adresse de Louvet, devenu le plus proche allié politique de Chénier (Lettre de J.-J. Dussault à J.-B. Louvet, député à la Convention nationale, au sujet de son journal, Paris, Maret, an III).

(31) Gallais, dans la Quotidienne du 23 messidor an III, exempte les ex-girondins de toute responsabilité dans les menaces qui pèsent sur la liberté de la presse. Il appelle à « distinguer la masse pure de la Convention de quelques membres pourris de ses comités ». 
par la résurrection littéraire d'André quelques mois plus tôt ${ }^{32}$, était une cible de choix : à la fois vulnérable sur le plan personnel et exemplaire de la continuité d'esprit avec l'an II que l'on imputait aux thermidoriens fidèles à la République.

\section{Un poète au tribunal de l'opinion}

Un nouveau périodique va s'emparer du sujet et lui donner une tout autre ampleur. Dans leurs numéros des 4 et 6 floréal, les Annales patriotiques et littéraires de Louis-Sébastien Mercier s'alarment du retour de l'arbitraire que manifestent à la fois l'arrestation de Lefortier et les déclamations contre le royalisme proférées par ceux qui autrefois « criaient contre le fédéralisme $»^{33}$. Seul responsable nommément désigné, Chénier est pris à parti avec une rare violence :

«On a remarqué que la persécution contre la liberté de la presse datoit de l'entrée de Chénier au comité de sûreté générale. L'esprit de vengeance qui, bien plus que l'amour du bien public, a fait précipiter dans les cachots le journaliste réclamé par l'Orateur du peuple, nous fait un devoir de signaler ce nouvel inquisiteur. On se rappelle en effet que Fortier [sic] avoit eu le tort de jeter quelques ridicules sur le risible auteur de Timoléon et autres niaiseries tragiques, sur ce misérable qui, après avoir lâchement laissé traîner à l'échafaud un frère qui valoit mieux que lui, n'a pas craint d'exposer aux yeux du peuple le spectacle de la discorde entre deux frères, de rappeler à notre souvenir le nom d'un fratricide au moment où André Chénier expiroit par la main du bourreau ? Ah ! sans doute si les septembriseurs eussent pu faire tous ces rapprochemens, un cri unanime d'indignation se seroit fait entendre contre ce misérable : Caïn qu'as-tu fait de ton frère? ».

(32) La Jeune Captive avait été publiée dans la Décade du 20 nivôse an III (9 janvier 1795).

(33) Annales patriotiques et littéraires, $n^{\circ}$ CXXVI, 6 floréal an III. Il se peut que Mercier plaide sa propre cause. Deux mois plus tôt, il avait été dénoncé comme « royaliste » au Comité de sûreté générale (voir les documents cités dans Louis-Sébastien MERCIER, Le Nouveau Paris, Jean-Claude BonNeT (éd.), Paris, Mercure de France, 1994, p. L-LI). Il n'a probablement pas oublié non plus le silence gardé par Chénier après les journées des 31 mai et 2 juin 1793, quand lui-même protestait contre la proscription de leurs collègues girondins, au prix de plusieurs mois d'emprisonnement. À noter qu'Ange Pitou, futur agent de Louis XVIII, tenait la chronique des séances de la Convention dans les Annales patriotiques et littéraires depuis fin germinal an III, en même temps qu'à l'instigation de Mercier, il rédigeait l'Ami du peuple de l'ancien jacobin Lebois dans le but de discréditer la cause révolutionnaire (Fernand ENGERAND, Ange Pitou, agent royaliste et chanteur des rues, Paris, E. Leroux, 1899, p. 83-87). Il n'est donc pas exclu que l'article sur Chénier soit une provocation issue de sa plume. 
En quelques lignes, tout est mis en place de l'accusation qui va poursuivre Chénier durant des années, sa vie entière peut-être, et au-delà même (il est possible que les Annales patriotiques reprennent des bruits répandus depuis un certain temps, peut-être justement par la Correspondance politique). Jusqu'à la formule finale du « caïnisme », qui fait évidemment écho à la fraternité révolutionnaire, devenue l'une des cibles de la réaction. La période jacobine y voyait l'expression d'un lien social renouvelé qui passait par la reconstruction des rapports sensibles (tout comme cela transparaît du reste dans le Timoléon de Chénier). Rendu suspect par son usage politique, le mot disparait aussi bien des formules officielles que des débats publics au cours de ce même printemps $1795^{34}$. Dans son rapport sur le renouvellement de la Convention, présenté le $1^{\text {er }}$ fructidor an III, Baudin déclarera sous les applaudissements : « Longtemps nous avons mis en affiche à la porte de nos maisons la fraternité, qui n'était alors que celle de Caïn et Abel. ${ }^{35}$. On sait que le mot est attribué à Chamfort, comme Mercier le rappelle en 1798 dans le chapitre « Fraternité » du Nouveau Paris. Mais la référence à Caïn renvoie également à une réactualisation propre au théâtre révolutionnaire ${ }^{36}$. La citation de la Genèse lancée contre Chénier avait été popularisée par La Mort d'Abel de Gabriel Legouvé, tragédie créée au Théâtre-Français en mars 1792. On y entendait Dieu lui-même adresser à Caïn la question fatidique, et l'intervention de la personne divine n'avait pas manqué de frapper les contemporains. Bien que la pièce de Legouvé fût a priori dénuée d'allusions contextuelles, la phrase s'était vue rapidement mise en rapport avec la situation de la France ${ }^{37}$, pour opposer ce « premier cri de la morale $»^{38}$ aux exactions commises au nom de la Révolution. Dès le printemps de l'an III, le thème de la fraternité ne cessera plus d'être tourné en dérision dans les écrits hostiles à la Convention, et en particulier par les adversaires de Chénier, auxquels il sert de prétexte pour accuser le député de fratricide à l'égard d'André, la violence symbolique de Timoléon devenant une réalité effective, du moins

(34) Marcel DAVID, Fraternité et Révolution française (1789-1799), Paris, Aubier, 1987, p. 166-175 ; Mona Ozouf, «La Révolution française et l'idée de fraternité », dans L'Homme régénéré. Essais sur la Révolution française, Paris, Gallimard, 1989, p. 177-180.

(35) Moniteur, 6 fructidor an III.

(36) Voir Pierre FRANTZ, « La fraternité hérö̈que dans le théâtre républicain de la Révolution », Cahiers parisiens/Parisian Notebooks, University of Chicago, 2007, p. 414-428.

(37) Esprit des journaux français et étrangers, vingt-troisième année, t. II, février 1794, p. 144

(38) Les derniers régicides ou Madame Élizabeth de France et Louis XVII, par M. le Chev. De M..., Londres, J. de Boffe, 1796, p. 47. 
sur le plan moral ${ }^{39}$. En ce sens, la campagne contre Chénier a pu être perçue comme une occasion de choix pour délégitimer ouvertement ${ }^{40}$ un sentiment emblématique de la socialité jacobine et sans-culotte.

La suite de l'article des Annales patriotiques et littéraires vise le promoteur de l'instruction publique. Mercier dénonce les images de la liberté «multipliées jusqu'au ridicule », avant de qualifier Chénier de «fondateur de secte » et de « second Mahomet » (comme on l'avait dit à propos de Robespierre) pour le rôle qu'il avait joué au Comité d'instruction publique dans l'organisation des fêtes décadaires. La polémique déclenchée par Mercier sera décisive également sous cet aspect. Au-delà de Chénier lui-même et du décret sur la censure, la presse mettra en procès l'idée d'une littérature et d'un art régénérés par la Révolution ${ }^{41}$, tout comme celle d'esprit public, dont on dénonce le caractère tyrannique, chargée qu'elle est des remugles du jacobinisme. Ironie du sort, c'est précisément cet esprit que Chénier entendait préserver avec les mesures votées le 12 floréal. Elles n'auront fait qu'en précipiter la chute. Dès le jour suivant, les lignes accusatrices de Mercier sont reproduites intégralement dans le Messager $d u$ soir de l'ex-jacobin Langlois et la calomnie poursuivra son chemin ${ }^{42}$.

Tout est donc prêt pour l'explosion générale qui va suivre le discours de Chénier. Pourtant, au lendemain du 12 floréal, les rapports de police n'enregistrent guère de mécontentement parmi la foule réunie au Jardin national. Au contraire : «Le décret rendu sur le rapport du citoyen Chénier a été [...] bien vu du public, en ce qui concerne les émigrés, le culte et le désarmement. Les esprits ont paru assez calmes ${ }^{43}$. La préoccupation de la population va surtout aux problèmes de ravitaillement. L'assaut viendra d'ailleurs. À lire les périodiques parisiens des jours suivants,

(39) On peut citer en particulier une satire de Joseph Michaud, publiée début 1797 : «Le grand Timoléon vint apprendre aux Français/ Que la fraternité n'était qu'une chimère,/ Et qu'on pouvait sans crime assassiner son frère. » (Petite dispute entre deux grands hommes, Paris, an V, p. 10).

(40) En dépit d'une teinte d'ironie, la première strophe du Réveil du peuple (« Peuple français, peuple de frères »), hymne de la contre-Révolution, maintenait une certaine ambiguïté.

(41) Ce n'est sans doute pas un hasard si Lefortier est également un amateur de belles-lettres, prompt à dénoncer leur recul dans la France révolutionnaire. Il publiera en 1799 un Aperçu sur les causes des progrès et de la décadence de l'art dramatique en France.

(42) Joseph Michaud, principal rédacteur de la Quotidienne à partir de l'été 1795, reconnaîtra n'avoir jamais cru aux accusations lancées contre Chénier. Il s'en serait servi pour décrédibiliser un adversaire influent (Antoine-Vincent ARNAULT, Souvenirs d'un sexagénaire, Raymond TROUSSON (éd.), Paris, Champion, 2003, p. 792). Lacretelle jeune n'accordait lui non plus aucun crédit aux rumeurs de fratricide (Charles de LACRETELLE, Histoire du Directoire exécutif, Paris, Treuttel et Würtz, 1826, t. II, p. 6). Selon Pierre Larousse, Roederer en parlait à la fin de sa vie comme de « la plus grande injustice de l'histoire de la Révolution » (Grand dictionnaire universel du XIX ${ }^{e}$ siècle, Paris, Administration du Grand dictionnaire universel, 1866-1877, t. IV, p. 14).

(43) Alphonse AULARD, Paris pendant la réaction thermidorienne et sous le Directoire, Paris, Cerf et Noblet, 1898-1902, t. I, p. 695. 
on croirait qu'une nouvelle Terreur est sur le point de s'abattre sur la France (le rapport de Chénier s'ajoutait, il est vrai, aux propositions de Thibaudeau pour renforcer le pouvoir du Comité de salut public ${ }^{44}$ ). La quasi-totalité des titres s'accorde à contester le décret sur la liberté de la presse, dont les critères plutôt vagues font craindre qu'il en soit fait un usage arbitraire ${ }^{45}$. Même le rival heureux de Chénier, Théodore Desorgues, choisi par Robespierre pour composer les paroles de l'hymne à l'Être suprême, lance contre lui une épigramme réprobatrice ${ }^{46}$. Mais seuls les journaux hostiles à la Révolution vont faire de l'épisode le point de départ d'un combat proprement politique. N'étaient-ils pas du reste les premiers concernés par le rapport ? On craint avant tout que la nouvelle loi ne confère à la Convention une autorité sans frein, alors que la fragilité du retour de la liberté et les incertitudes de la justice thermidorienne rendent le contre-pouvoir de la presse plus indispensable que jamais ${ }^{47}$. En punissant la réflexion critique au même titre qu'une incitation à l'émeute, elle entravera le légitime exercice des lumières sur les limites du pouvoir politique ${ }^{48}$. La Convention a pris ainsi le risque de revenir «à la persécution des mots » en vigueur sous Robespierre ${ }^{49}$ et de « travestir la liberté en superstition politique $»^{50}$ :

« Nous avons renversé le tyran, nous avons voué à l'exécration des siècles et ses ministres et leurs fureurs ; \& c'est après 8 mois d'intervalle, nos plaies encore saignantes qu'un homme né sur les rives du Bosphore vient nous ressasser ces vieilles idées, et ose nous frapper de cet épouvantail usé ! » ${ }^{51}$

C'est donc la fin du processus enclenché le 9 Thermidor qu'il faut redouter, et un retour de la Terreur sous une forme plus policée : «[...] Que Chénier s'apprête à signer en prose l'exil de tous les départements qui ont dénoncé Carrier, Maignet, Lecarpentier. Robespierre régna sur des

(44) La Quotidienne, 15 floréal an III.

(45) Y compris le Moniteur (18 floréal an III), habituellement proche du gouvernement. Alma Söderhjelm signale également quelques brochures qui se bornent à défendre la liberté de la presse sans arrière-pensées politiques, Alma SÖDERHJELM, op. cit., t. II, p. 40-42. Pour les périodiques d'inspiration jacobine, voir la réaction négative du Journal des hommes libres (15 floréal an III). Par contre, le Journal universel fait paraître un compte rendu fort laudateur dans son numéro du 13 floréal.

(46) Journal de Paris, 25 floréal an III.

(47) L'Orateur du peuple, 14 et 18 floréal an III ; La Quotidienne, 16 floréal an III ; Nouvelles politiques nationales et étrangères, 16 floréal an III.

(48) La Quotidienne, 16 floréal an III.

(49) Le Courrier républicain, 14 floréal an III.

(50) La Quotidienne, 17 floréal an III.

(51) Ibidem, 16 floréal an III (Chénier était né à Constantinople). 
cadavres, Chénier régnera sur des déserts $»^{52}$. On taxe cet $«$ inquisiteur $»^{53}$ de « nouveau David $»^{54}$ ou on l'affuble du sobriquet d' « Omar $»^{55}$, réservé à Robespierre quelques mois plus tôt. Si un soutien de façade à la République est souvent affiché, les journaux sont unanimes pour réfuter tout risque de retour en force du royalisme dans l'opinion ${ }^{56}$, quitte à dénier aux écrits " séditieux » une véritable influence sur les esprits ${ }^{57}$. En réalité, les écrivains hostiles à la Révolution saisissent l'occasion pour renforcer leur liberté de parole, en abandonnant de plus en plus toute apparence de respect à l'égard de la République ${ }^{58}$. C'est que certains pointent les divisions à l'œuvre chez ses partisans eux-mêmes ${ }^{59}$.

La presse contre-révolutionnaire ne s'arrête pas à ces critiques générales. Au fil des articles prend forme une véritable campagne dirigée cette fois contre la personne de Chénier, considéré à la lumière de l'affaire Lefortier comme le principal responsable de la loi du 12 floréal et du redécoupage politique qu'elle impose. Qui est vraiment ce député qui ambitionne de devenir «le Washington de la République » après s'en être cru le poète tragique ${ }^{60}$ ? On a beau jeu de rappeler les liens qu'il a entretenus avec quelques membres peu recommandables du régime montagnard, comme David ${ }^{61}$ ou encore Dupin ${ }^{62}$, inquiété au même moment pour son rôle dans l'exécution des fermiers-généraux. Exilé à Londres d'où il commente les événements parisiens pour le public des émigrés, Jean-Gabriel Peltier ne s'embarrasse pas des précautions qui modèrent quelque peu ses confrères du continent : Chénier, décrit comme l' « auteur dramatique à qui l'on doit la majeure partie des tragédies révolutionnaires qui ont été représentées depuis cinq ans », s'est "rendu coupable au 10 août et au 2 septembre 1792, de plusieurs de ces traits de faction et de cruauté qui fondaient alors le titre d'un homme au patriotisme et ses droits

(52) Le Messager du soir, 14 floréal an III. Bien que plus nuancées, les Annales patriotiques et littéraires ne s'inquiètent pas moins : « [...] Le rapport de Chénier n'est-il pas un cadre où demain, peut-être, viendra se placer un second Robespierre? » (15 floréal an III).

(53) La Quotidienne, 15 floréal an III.

(54) Le Messager du soir, 12 floréal an III.

(55) L'Orateur du peuple, 22 floréal an III.

(56) Voir notamment L'Orateur du peuple, 18 floréal an III.

(57) La Quotidienne, 17 floréal an III.

(58) Voir les Nouvelles politiques nationales et étrangères, 28 floréal an III.

(59) Le Messager du soir, 28 floréal an III.

(60) La Quotidienne, 20 floréal an III.

(61) L'Orateur du peuple, 18 floréal an III.

(62) La Quotidienne, 26 messidor an III. 
au pouvoir législatif $»^{63}$. Surtout, la mort d'André est rappelée avec une cruelle ironie. Lacretelle jeune, qui a connu et admiré André Chénier au club des Feuillants, demande au rapporteur de la loi du 12 floréal de faire appel à sa mémoire, qu'il semble avoir bien courte :

«Chénier n'entend-il pas quelquefois les mânes plaintifs de tant de victimes égorgées sous [1'] affreux prétexte [de royalisme]... Chénier, pourquoi le tableau que je te présente, te fait-il détourner les yeux ? Crainstu de reconnaître parmi ces victimes ton frère éloquent, égorgé par les décemvirs, comme écrivain royaliste ? ${ }^{64}$

Qu'a donc tenté Chénier pour sauver de la guillotine ce frère si bon poète ${ }^{65}$, lui qui est venu au secours d'un David quelques mois plus tôt ${ }^{66}$ ? Cela lui vaut des jeux de mots cinglants sur la soi-disant fraternité des Républicains ${ }^{67}$. On prétend, rapporte un journal, qu'il « reçoit tous les matins une lettre de la même main, qui ne contient que cette interrogation terrible : qu'as-tu fait de ton frère ? $\gg^{68}$. Derrière les vertus politiques qu'il affiche, se dissimule l'amour-propre d'un auteur dramatique prêt à tout pour défendre son art. Ne s'est-il pas montré plus inquiet du sort d'une de ses pièces que de celui d'André Chénier ${ }^{69}$ ?

Car c'est le poète qui est, en fin de compte, la cible favorite de toutes les attaques. La politique n'est pour lui qu'un prétexte, puisque son rapport du 12 floréal «n'a été inspiré que par le désir d'exercer une sorte de dictature sur les théâtres et la littérature $\gg^{70}$, pour s'assurer une gloire que ses pièces ne lui mériteront pas : «Qu'un homme né aux pieds des 7 tours veuille y faire enfermer, son frère, son ami, parce qu'il aura ri à ses tragédies, ou dormi à ses capucinades, cela se conçoit, cela ne nous surprend point $»^{71}$. Ou encore :

(63) Paris pendant l'année 1795, Londres, 1795, t. I, p. 87. Pour les accusations montées contre Chénier sur son rôle présumé dans les Massacres de Septembre, voir Alfred J. BINGHAM, op. cit., p. $98-100$.

(64) Nouvelles politiques nationales et étrangères, 16 floréal an III.

(65) Le Courrier républicain, 15 floréal an III ; La Quotidienne, 15 et 17 floréal an III.

(66) La Quotidienne, 17 floréal an III.

(67) L'Orateur du peuple, $1^{\text {er }}$ floréal an III ; Le Messager du soir, 13 floréal an III.

(68) Le Messager du soir, 17 floréal an III. Arnault rapporte que la Quotidienne répétait cette question à chaque numéro (« Lettre sur M.-J. Chénier », dans CEuvres de M.-J. Chénier, Paris, Guillaume, 1824-1826, t. I, p. XV et Souvenirs d'un sexagénaire, op. cit., p. 792), mais il n'en est aucune trace, même si le périodique se montra bien l'un des plus virulents contre Chénier à partir de 1796.

(69) Le Messager du soir, 17 floréal an III.

(70) Jean-Gabriel PELTIER, op. cit., t. I, p. 89.

(71) La Quotidienne, 15 floréal an III. 
«La grande haine de Chénier contre [les émigrés, les prêtres réfractaires et les journalistes royalistes], vient des coups de sifflets, ou de censures qu'il en a éprouvés. Sa vanité est sa seule règle ; sa république n'est que dans le parterre des spectacles; il regarde tous ses auditeurs comme ses ennemis. Telle est la politique de Chénier [...]. » ${ }^{72}$

« Mauvais poète $»^{73}$, lâche et orgueilleux ${ }^{74}$, il berne son auditoire en usant de grands mots, comme il l'a fait à la Convention pour présenter sa loi. Le « petit poète lauréat de la République ${ }^{75}-$ ainsi que le qualifie Peltier en faisant allusion au poète attaché à la cour d'Angleterre est le porte-drapeau des prétentions d'une Révolution en réalité à bout de souffle $\mathrm{e}^{76}$, tout juste bonne à s'envelopper d'un pathos autoritaire : «Bientôt il nous sera peut-être enjoint, pour ne pas avilir la représentation nationale dans l'un de ses membres, de préférer aux chefs-d'œuvre de Racine, le pathos de Chénier, vu que le premier était un esclave et que le second est le représentant d'un peuple libre $\gg{ }^{77}$. On a le sentiment de voir brusquement éclater toutes les inimitiés, politiques et personnelles, qui s'étaient accumulées autour de Chénier depuis 1789 et que la rupture de Thermidor avait jusque-là détournées. Le 26 floréal, le Messager du soir dresse en ces termes l'épitaphe de «Chénier-Pradon »: «Ci-gît/Le poète Chénier qui fit/Primidi,/Duodi,/Tridi, etc./C'est tout ce que l'histoire en dit. »- se souvenant sans doute de son rôle dans la création du calendrier révolutionnaire, enterré dans le même geste. En l'espace de quelques semaines, l'image du « poète de la Révolution » est irrémédiablement ternie. Sa participation à la culture montagnarde est volontairement présentée sans les nuances qu'il avait voulu y apporter, afin d'en donner une version radicale : «Sublime auteur des sans-culottides, digne successeur de [...] Saint-Just, grand pontife de l'Être-Suprême, chantre immortel de [la] constitution montagnarde ${ }^{78}$, grand panégyriste de Marat, apôtre de Robespierre ${ }^{79}$. Les deux derniers griefs étaient sans fondement, mais le reste des accusations rappelait le rôle central de Chénier dans les cérémonies

(72) Ibidem, p. 88.

(73) Le Courrier républicain, 15 floréal an III.

(74) L'Orateur du peuple, 18 floréal an III.

(75) Jean-Gabriel PELTIER, op. cit., t. I, p. 87.

(76) La Quotidienne, 29 messidor an III.

(77) Le Messager du soir, 14 floréal an III.

(78) Des vers de Chénier avaient bien été chantés à la Convention le 14 juillet 1793 pour la proclamation de la nouvelle constitution (Moniteur, 17 juillet 1793), mais il s'agissait des dernières strophes de son Chant du 14 juillet, écrit pour la Fête de la Fédération de 1790, et non d'un hymne « montagnard » composé à cette occasion.

(79) Le Messager du soir, 28 floréal an III. 
de l'an II. Il est devenu, pour une partie de l'opinion, le terroriste qu'il n’avait jamais été.

\section{Les héritiers des Philosophes contre la République}

Trois réfutations plus développées paraissent au cours des mêmes semaines. Elles proviennent de personnalités célèbres aux parcours politiques divers, réunies par une hostilité commune contre le nouveau républicanisme de la Convention : La Harpe, Morellet et Roederer. Leur publication semble du reste avoir été concertée ${ }^{80}$. Passons sur la première ${ }^{81}$, dont le propos est essentiellement politique. Les deux autres substituent aux invectives de la presse une critique plus large des ambitions de Chénier en matière d'instruction publique, à travers ce qui en fut pour lui l'expression privilégiée, à savoir le théâtre.

Après avoir traversé la Terreur dans le plus grand désarroi ${ }^{82}$, l'abbé Morellet n'a pas tardé à reprendre ses activités de publiciste en Thermidor, rédigeant coup sur coup deux pamphlets où il défend les droits des victimes de la période jacobine (Le Cri des familles, en décembre 1794 et La Cause des pères, en mars 1795). La loi présentée par Chénier le 12 floréal est l'occasion d'un nouvel imprimé, sur un sujet dont Morellet s'était fait le champion sous l'Ancien Régime, après en avoir été brièvement le martyr ${ }^{83}$ : les Pensées libres sur la liberté de la presse, à l'occasion d'un rapport $d u$ représentant Chénier à la Convention nationale, du 12 floréal. Tout comme celui de La Harpe, le texte de Morellet a été écrit très vite (il porte la date du 16 mai); il est le fruit d'une réaction épidermique qui traduit sans doute une certaine alarme. Mais contrairement à l'auteur du Lycée, Morellet s'exprime en homme des Lumières, ce qui lui donne plus d'autorité : son pamphlet tente de se réapproprier leur héritage, hautement revendiqué par la République thermidorienne. Derrière cette polémique se

(80) C'est ce que suggèrent l'allusion à Roederer sur laquelle s'ouvre la brochure de La Harpe et une lettre de Morellet au premier du 26 floréal an III (15 mai 1795) : « Jamais le maître Pythagore ou le maître Quesnay n'ont été loués par leurs disciples aussi bien que vous me loués. Ma modestie vous pardonne parce que vos eloges tournent au profit des bonnes causes que nous defendons vous et moi et mon sentiment vous en remercie. Je crois maintenant que le Chenier en a assés. Il faut revenir à nos moutons qu'on egorge [,] je veux dire aux peres et meres d'emigrés. » (Lettres d'André Morellet, Dorothy MEDLIN et Jean-Claude DAVID (éd.), t. II, Oxford, The Voltaire Foundation, 1994, p. 211).

(81) La Liberté de la presse défendue par La Harpe contre Chénier, Paris, Migneret, an III.

(82) Comme il le raconte au ch. XXV de ses Mémoires sur le XVIII e siècle et sur la Révolution.

(83) Morellet avait été incarcéré à la Bastille durant six semaines en 1760 à cause d'une satire contre Les Philosophes de Palissot, dans laquelle il avait eu le tort de s'en prendre à la protectrice du dramaturge, la princesse de Robecq, maîtresse de Choiseul. Il avait publié en 1775 des Réflexions sur les avantages de la liberté d'écrire sur les matières d'administration. 
devine également l'affrontement de deux générations d'hommes de lettres : celle des académiciens de l'Ancien Régime, balayée par la Révolution, et celle des littérateurs issus de $1789^{84}$. Le conflit est particulièrement net dans le cas présent. Il se cristallisera à l'Institut, puis à l'Académie reformée en 1803 par les soins de Suard et Morellet. Les Mémoires de Morellet, qui reproduisent en annexe les Pensées libres sur la liberté de la presse de l'an III, accréditeront l'idée que Chénier a pris part aux crimes de la Terreur, au même titre qu'un Tallien ${ }^{85}$. Or ce genre d'accusation infamante, sous la plume d'une personnalité comme Morellet, affectera la réputation de Chénier aux yeux de la postérité immédiate, celle de la Restauration ${ }^{86}$, contribuant ainsi à noircir son image auprès d'un $\mathrm{XIX}^{\mathrm{e}}$ siècle anti-jacobin.

Morellet rappelle dès les premières lignes du pamphlet son appartenance historique au parti des Philosophes, laissant entendre qu'il a plus que quiconque le droit d'évoquer les combats auxquels ils ont attaché leur nom. N'a-t-il pas connu les cachots de l'Ancien Régime précisément pour avoir fait un usage trop libre de sa plume ? Il a pu constater alors l'état de tolérance dont on jouissait malgré tout sous le «despotisme » de la monarchie, à l'opposé des censures en tous genres auxquels la Révolution n'a que trop habitué les esprits. Comment supporter la limitation que le gouvernement révolutionnaire entend donner aujourd'hui à la liberté d'opinion, après avoir mis fin à la tyrannie ? Un interlocuteur imaginaire a vite fait de rassurer l'abbé : il est impossible que la nation retrouve l'oppression des deux années précédentes, pour la bonne raison qu'on n'attrape pas deux fois la même maladie. Mieux vaut donc « rire de Chénier, au lieu d'en avoir peur » et « de prendre la chose au tragique $»^{87}$. Partant, Morellet n'est guère en peine de montrer le « ridicule » des soi-disant délits sanctionnés par la nouvelle loi. Son raisonnement est l'occasion d'une allusion désobligeante

(84) En revenant le 6 messidor an III sur les attaques dirigées contre lui, Chénier parlera d' « écrivains aguerris depuis soixante ans à l'esclavage et à la nullité » (Euvres de M.-J. Chénier, op. cit., t. V, p. 266). Le décalage ressenti par Morellet devant la culture révolutionnaire a été mis en évidence par Robert Darnton («Un philosophe face à la Terreur », dans Gens de lettres, gens du livre, Paris, Odile Jacob, 1992, p. 139-150).

(85) Morellet évoque, à propos du soulèvement royaliste de vendémiaire an IV, « le crédit bientôt rendu aux membres de la Convention qui avaient participé aux crimes du gouvernement révolutionnaire, tels que Tallien, Louvet, Chénier, etc. » (Mémoires de l'abbé Morellet sur le XVIII siècle et sur la Révolution, Paris, Ladvocat, 1821, t. II, p. 152).

(86) L'épouse de Joseph-Marie de Gérando écrira à son fils le 31 juillet 1821, à la parution des Mémoires de Morellet, que ceux-ci sont lus « avec avidité » dans la société parisienne (Lettres de la baronne de Gérando, Paris, Didier et $\mathrm{C}^{\mathrm{ie}}, 1880$, p. 358).

(87) Pensées libres sur la liberté de la presse, à l'occasion d'un rapport du représentant Chénier à la Convention nationale, du 12 floréal, Paris, Maret, an III, p. 9. 
au « fratricide $»^{88}$, par laquelle il rappelle à Chénier que son frère avait perdu la vie pour ses articles contre les jacobins ${ }^{89}$.

La brochure s'est prend également à un point resté un peu dans l'ombre jusqu'ici, l'article sur la direction de l'esprit public qui concluait la loi du 12 floréal. Morellet ne se contente pas de dénoncer le risque de nouvelles mesures autoritaires. C'est tout simplement l'idée d'instruction publique qui est visée :

«La liberté, la République sont, sans doute, de fort bonnes choses, mais je déclare que si l'on veut m'en parler et me les montrer sans cesse, et partout, on me les fera prendre en grippe, et on m'ennuiera à la mort, et pour que nous puissions aimer la République et la liberté, il ne faut pas qu'elles nous ennuient $»^{90}$.

À sa manière, la critique de Morellet équivaut sur le plan des lettres et des arts à la distinction que Benjamin Constant établira pour la politique une décennie plus tard en opposant la liberté des modernes à celles des anciens ${ }^{91}$, à savoir une émancipation hors du legs révolutionnaire. Elle signe ainsi une véritable crise culturelle (ou un retour à l'ordre des choses, si l'on préfère), qui voit les modes d'expression inventés par les hommes de 1789-1794 plonger dans un état d'agonie. Morellet s'attaque à l'idéal républicain, dont il raille la prétention à régenter la totalité des productions artistiques : « Les auteurs dramatiques ne feront-ils plus que des Caïus Gracchus, des Charles IX, des Timoléon, et les peintres et sculpteurs, que des J.-J. Rousseau, des Mirabeau, ou même des Brissot et des Marat ? $\gg^{92}$. Les beaux-arts n'ont qu'un seul but « immédiat et principal », l'imitation. S'ils peuvent de plus en avoir un second de nature morale, il « est absolument distinct de ce but purement politique auquel Chénier veut qu'ils soient dirigés $»^{93}$. L'œuvre du poète-député n'en offre-t-elle pas la meilleure illustration ? « [...] S'il eût sauvé André Chénier, que pleurent les lettres et l'amitié, et qu'il l'eût sauvé en bravant de grands dangers, ou même en y succombant, c'eût

(88) «Sultan Chénier, né à Constantinople, en auriez-vous rapporté les mœurs des Ottomans, qui croient ne pouvoir régner qu'en étranglant leurs frères ? (ibidem, p. 10).

(89) Morellet avait connu André Chénier chez les frères Trudaine et l'avait fréquenté durant la Révolution (Dorothy MEDLIN, « André Morellet and the Trudaines », Studies on Voltaire and the Eighteenth Century, Oxford, The Voltaire Foundation, 2005, n ${ }^{\circ} 2$, p. 117-125).

(90) Ibidem, p. 14.

(91) Distinction en germe au cours des mêmes mois de 1795, comme Marcel Gauchet l'a montré (La Révolution des pouvoirs. La souveraineté, le peuple et la représentation (1789-1799), Paris, Gallimard, 1995, p. 181-182).

(92) Ibidem, p. 13.

(93) Ibid., p. 14-15. 
été là un sujet de tragédie, plus beau, à mon sens, et bien plus moral que Timoléon ${ }^{94}$. C'était refuser à la tragédie toute distance par rapport aux événements et, par-là, nier sa capacité critique à les interpréter sans pour autant s'y brûler les ailes. Morellet réduit donc de manière décisive la portée politique des lettres, que Chénier avait mise au centre de son esthétique avant même le bouleversement de 1789, précisément par le biais de la morale. Et il inscrit ce rabaissement dans l'intimité biographique de l'auteur de Timoléon, dont la pièce accomplissait - volontairement ou pas ce divorce de la politique d'avec la morale.

Venons-en au troisième protagoniste, tapi en embuscade derrière les réponses de La Harpe et Morellet, à savoir Roederer. L'ex-procureur syndic du département de la Seine, revenu à la vie politique après une éclipse volontaire sous la Terreur, est une des figures clés de la période thermidorienne. Proche de personnalités aussi importantes que Tallien et Merlin de Thionville, dont il a composé certains discours ${ }^{95}$, il est de ceux qui brouillent la frontière entre réaction anti-jacobine et désir d'en finir tout bonnement avec l'héritage de la Convention. Dès l'automne 1794, Roederer participe aux campagnes d'opinion conduites par les anciens monarchistes constitutionnels (dont Morellet), d'abord à travers quelques brochures anonymes puis dans les colonnes du Journal de Paris, dont il devient co-propriétaire en janvier $1795^{96}$. Il ne pouvait que réagir très vivement à la nouvelle impulsion « révolutionnaire » donnée à la politique des Comités, et en particulier à la loi du 12 floréal. Bien d'autres républicains modérés étaient sans doute dans son cas, à l'image du conventionnel Baudin ${ }^{97}$, dont le Journal de Paris publie un échange de lettres avec Roederer en faveur de la liberté de la presse (sur sept numéros, du 14 au 22 floréal an III $^{98}$, Roederer poursuivant seul leur réflexion les 23, 25 et 27 floréal).

La discussion adopte un ton en apparence fort respectueux à l'égard de Chénier. Mais elle illustre à la fois l'incompréhension provoquée par son décret sur la presse dans une large fraction de l'opinion et les contradictions

(94) André MORELLET, op. cit., p. 12.

(95) Kenneth Margerison, P.-L. Roederer : Political Thought and Practice during the French Revolution, Philadelphie, Transactions of the American Philosophical Society, vol. 73, 1983, p. 114-115.

(96) Voir Thierry LENTZ, «La presse républicaine modérée sous la Convention thermidorienne et le Directoire : Pierre-Louis Roederer, animateur et propriétaire du Journal de Paris et du Journal d'Économie publique », Revue historique, n592/2, octobre-décembre 1994, p. 297-313.

(97) Pierre Baudin, député des Ardennes, avait été proche des girondins, et en particulier de Louvet. En Thermidor, il avait participé activement à la déjacobinisation, avant de siéger dans la commission des Onze, chargée de préparer la nouvelle constitution. Il occupait donc une position influente.

(98) Roederer annonce sa publication en brochure, mais il semble y avoir finalement renoncé. 
à l'œuvre chez les thermidoriens. Il ravive notamment un clivage apparu quelques mois plus tôt lors des débats sur le rétablissement de la liberté de culte : chargé de l'organisation des fêtes décadaires en nivôse an III, Chénier était l'un des représentants éminents de ce qu'Aulard appelle «les "philosophes" de la Convention ${ }^{99}$, alors que Baudin et Roederer avaient au contraire défendu le retour de la religion. Craint-on au sein du camp des « républicains bourgeois » les prémices d'une contre-réaction « néo-montagnarde », qui va rapidement s'avérer toute relative en limitant son ambition à préserver le régime républicain ? Loin d'éclater au grand jour, l'adoption de la Constitution de l'an III occultera provisoirement ces tensions. Dans l'immédiat, le différend se révèle à travers la volonté affichée par les thermidoriens conservateurs de rompre avec les références issues de la Révolution - auxquelles Roederer ne cesse de s'en prendre dans le Journal de Paris, ironisant sur l'abus des mots «fanatisme» et « royalisme » ou sur le congé décadaire. Le point essentiel est pourtant ailleurs. Les deux partis ont des conceptions difficilement conciliables de ce qui doit diriger la vie publique : l'Assemblée des élus ou l'opinion « modérée »? On le voit avec l'argument majeur que Roederer oppose à Chénier pour défendre la presse : affaiblie et désorientée après la perte de ses membres les plus talentueux, la Convention a plus que jamais besoin de la liberté d'analyse des publicistes « éclairés ». Eux seuls peuvent lui éviter de retomber dans ses anciennes ornières ${ }^{100}$.

L'échange entre Baudin et Roederer aborde enfin la question de l'usage politique du théâtre, prôné dans le rapport de Chénier. Le député des Ardennes a beau jeu de rappeler, avec malignité, que le sujet avait déjà été mis à l'ordre du jour par la Convention montagnarde : son décret du 2 août 1793, qui instaurait des spectacles « de par et pour le peuple », ne répondait-il pas au même souci de diriger l'esprit public ? Or il n'avait fait qu'accentuer la surenchère révolutionnaire, au point qu'un représentant en était arrivé à s'attaquer à l'une de ces pièces républicaines, parce qu'il la jugeait trop tiède ${ }^{101}$. Cette pièce, c'était le Caïus Gracchus de Chénier, dont les déboires auprès des autorités jacobines en octobre $1793^{102}$ sont

(99) Alphonse Aulard, Histoire politique de la Révolution française, Paris, Armand Colin, 1901, p. 538.

(100) Journal de Paris, 21 floréal an III. Sur le rôle de l'opinion dans les idées politiques de Roederer pendant le Directoire, voir Jean-Luc CHAPPEY, «Pierre-Louis Roederer et la presse sous le Directoire et le Consulat : l'opinion publique et les enjeux d'une politique éditoriale », $A H R F$, oct.-déc. 2003, n 334 , p. $1-21$.

(101) Journal de Paris, 21 floréal an III.

(102) Voir Adolphe LIÉBY, « La presse révolutionnaire et la censure théâtrale sous la Terreur », La Révolution française, t. 45, juillet-décembre 1903, p. 460-470. 
habilement retournés contre son auteur, pour prouver que le public ne se laisse pas si facilement diriger et qu'à force de vouloir le contrôler, on pourrait bien rappeler cet « esprit de vertige » apparu avec la Révolution. $\mathrm{Au}$ lieu de vouloir agir sur l'opinion, une prudence discrète suffirait pour éviter que les spectacles ne servent de prétexte à des manifestations royalistes : «Rapportez-vous-en au discernement des convenances de la part des acteurs, sans vous dispenser néanmoins de les surveiller, ce qui est le devoir des gouvernements sages ${ }^{103}$. Qu'on évite d'assujettir au pouvoir le monde des spectacles et ses petites rivalités en créant un théâtre au service de l'État. Et surtout, qu'on se garde bien d'établir une censure qui ne ferait que ressusciter en sens inverse celle de l'Ancien Régime : "Qu'on ne puisse pas répéter, toujours d'après Figaro, vous n'écrirez ni contre le culte (aujourd'hui il faudrait dire pour le culte), ni contre l'opéra, ni contre le gouvernement ${ }^{104}$. À ces arguments politiques vont s'en ajouter d'autres, d'ordre strictement dramatique cette fois. Trois jours plus tard, Roederer, en rendant compte de la brochure de Morellet contre Chénier, rappelle les idées sur la tragédie qu'il avait exposées dans le numéro du 24 germinal an III (13 avril 1795) à propos de Quintius Cincinnatus, pièce d'Antoine-Vincent Arnault :

« Nous avons observé, il y a quelque temps, que les intérêts domestiques pouvaient seuls fournir des sujets pathétiques à l'art du théâtre ; qu'avec des événements purement politiques, le poète ne pouvait produire que des tragédies sans tragique; et que chez les Grecs comme chez nous tous les spectateurs ont toujours été très froids aux drames de ce genre. Ainsi quand l'art dramatique serait dirigé uniquement vers l'affermissement de la République, il servirait très peu à la République. Il ne suffirait pas que les poètes se bornassent à faire des drames politiques; il faudrait encore contraindre les spectateurs à venir les entendre ; à quoi servirait de purifier le théâtre si on le faisait déserter? ${ }^{105}$

Roederer prolonge la critique esquissée par Morellet à coup de sarcasmes contre le théâtre patriotique ou ce qu'il en reste. Il indique aux auteurs tragiques la voie qu'ils privilégieront sous le Directoire, au moment où Chénier cessera précisément d'écrire pour la scène, en réservant son engagement à la satire, comme on le lui conseille déjà ici. Mais ce sera 
au détriment de Roederer lui-même ${ }^{106}$ car les positions politiques des uns et des autres, en se dévoilant progressivement, n'auront fait qu'aiguiser l'affrontement au sein de l'ex-camp thermidorien.

Ainsi lancée, la campagne contre Chénier ne connaîtra guère de trêve jusqu'à la fin du Directoire. Avec de nouvelles salves, d'abord au printemps 1796, lorsque reprendra le débat sur la censure, puis durant l'hiver 1796-1797, après la publication du Discours sur la calomnie, où Chénier se défendra enfin. Une différence de taille la sépare pourtant de celles qui l'ont précédée sous la Révolution : c'est une guerre de papier, qui ne fera pas verser une goutte de sang, et l'adversité politique n'exclut pas toujours l'estime sur le plan personnel ou littéraire ${ }^{107}$. L'animosité, même si elle ressemble parfois à de la haine, semble s'être limitée à des échanges de libelles, sans jamais remettre en cause la frontière fragile qui sépare l'écrit de la violence. Comme s'il importait à tout prix de ne pas emprunter les chemins qui avaient mené à la Terreur.

Renvoyée au Comité de législation, la loi sur la presse du 12 floréal y restera enterrée jusqu'à la fin de la Convention. Les périodiques hostiles à la République ne se trouveront plus vraiment inquiétés, du moins jusqu'au soulèvement royaliste du 13 vendémiaire an IV, qui mobilisera les publicistes de la réaction. Mais l'état d'esprit a indéniablement changé : la Constitution de l'an III, signée le 5 fructidor, ne mentionne pas la liberté d'expression dans sa déclaration des droits fondamentaux (alors que la liste des devoirs demande de respecter ceux qui sont les « organes » des lois). Et si le texte constitutionnel en fournit bien la garantie, il prévoit également sa suspension en cas de troubles jusqu'à une durée d'un $a^{108}$. L'insurrection royaliste de Vendémiaire bouleversera la situation et plusieurs journaux se verront mis sous scellés. Devant l'audace recrudescente des périodiques d'opposition, la question ne tardera pas à être rouverte par le Directoire en l'an IV $^{109}$ (et Chénier s'y intéressera à nouveau), sans trouver de véritable solution jusqu'à l'arrêté consulaire du 27 nivôse an VIII, qui réduira la presse politique à treize titres agréés par le pouvoir ${ }^{110}$. Quant aux mesures préconisées par Chénier pour relever l'esprit public, elles ne

(106) Le Docteur Pancrace, Paris, Laran, an V.

(107) Voir Charles de LACRETELLE, Histoire du Directoire exécutif, op. cit., t. II, p. 5-7 et le témoignage d'Arnault à propos de Joseph Michaud (« Lettre sur M.-J. Chénier », dans CEuvres de M.-J. Chénier, op. cit., t. I, p. XV-XVI).

(108) Alma SÖDERHJELM, op. cit., t. II, p. 45-46.

(109) Ibidem.

(110) Roederer fera partie de la commission établie à cette fin. 
verront jamais le jour, mis à part deux décrets pris par la Convention les 26 et 28 messidor dans l'espoir de mettre un terme aux huées lancées contre les hymnes révolutionnaires et au succès toujours plus grand du Réveil du peuple. Étaient-elles encore d'actualité à l'heure où L'Intérieur des comités révolutionnaires, la féroce comédie satirique de Ducancel, triomphait au Théâtre de la Cité-Variétés?

Gauthier AMBRUS

Université Paris-Sorbonne

Centre d'Étude de la Langue et de la Littérature françaises

des $\mathrm{XVII}^{\mathrm{e}}$ et $\mathrm{XVIII}^{\mathrm{e}}$ siècles ambrusg@yahoo.fr 